\title{
Predicción mediante redes neuronales de la temperatura de arrabio de un horno alto. Temperatura subyacente de $\operatorname{arrabio}^{(\cdot)}$
}

\author{
C. Cantera*, J. Jiménez*, I. Varela*y A. Formoso*
}

\begin{abstract}
Resumen Basándose en un sencillo modelo termodinámico, se propone el criterio de temperatura subyacente como medio para estudiar la tendencia de temperatura de un horno alto. Como aplicación, se implementa una red neuronal que puede trabajar en tiempo real en una planta y hace una predicción válida de la temperatura de colada del arrabio con una antelación de 2 a 16 h, con una horquilla de error lógicamente creciente.
\end{abstract}

Palabras clave Horno alto. Temperatura arrabio. Redes neuronales. Predicción. Simulación.

\section{Hot metal temperature prediction by neural networks in the blast furnace}

\begin{abstract}
Based on a simplified model, the underlying temperature criteria is proposed as a method to study the temperature trends in a blast furnace. As an application, a neural network able to forecast hot metal temperatures from 2 to $16 \mathrm{~h}$ in advance (with decreasing precision) has been built. This neural network has been designed to work at real time in a production plant.
\end{abstract}

Keywords

Blast furnace. Hot metal temperature. Neural networks. Prediction. Simulation.

\section{INTRODUCCIÓN}

Un horno alto es una gran cuba donde, mediante la combustión de carbón de coque en presencia de mineral de hierro, se obtiene arrabio líquido que sale por la parte inferior a intervalos regulares llamados coladas. En la cuba, no solo se introducen componentes sólidos compactos, como los ya citados, sino pulverizados, como el carbón de coque, y gaseosos, como aire, oxígeno o vapor de agua. Estos últimos se introducen a presión por unas toberas especiales, razón por la cual se denominan "componentes de soplado".

La composición o calidad del arrabio depende, básicamente, de la temperatura de salida del arrabio líquido, temperatura que, a su vez, depende de los elementos citados arriba, así como del tiempo. Un exceso o defecto en la temperatura de salida sobre la temperatura ideal supone una pérdida de calidad del arrabio y, consecuentemente, un incre- mento en los costos del procesado posterior para obtener la calidad final deseada .

La predicción de la temperatura de salida del arrabio de un horno alto tiene una indudable utilidad, pues permite corregir los factores de desequilibrio antes de que éste se produzca.

Desde el punto de vista termodinámico, un horno alto es un sistema no cerrado en el que influyen numerosas variables, fundamentalmente los componentes introducidos (citados más arriba).

Dado el gran número de variables que influyen en la evolución del horno, el estudio analítico de este dispositivo es casi inviable. Sin embargo, las redes neuronales son una herramienta muy adecuada para el estudio de este tipo de problemas. En el presente trabajo se ha utilizado dicha herramienta para predecir la temperatura del arrabio líquido.

Como base de este estudio de predicción de temperatura, se utilizaron todos los datos de las variables que se registraron en un periodo de $69 \mathrm{~d}$ en

(•) Trabajo recibido el día 4 de octubre de 2000 y aceptado en su forma final el día 9 de julio de 2002.

$\left(^{*}\right)$ Centro Nacional de Investigaciones Metalúrgicas (CENIM), Consejo Superior de Investigaciones Científicas (CSIC), Avda. Gregorio del Amo, 8. 28040 Madrid (España). 
Predicción mediante redes neuronales de la temperatura de arrabio de un horno alto. Temperatura subyacente de arrabio

C. CANTERA, J. JimÉNEZ, I. VARELA y A. Formoso

el horno alto B de Gijón, perteneciente a la empresa Aceralia .

La mayoría de las variables fueron muestreadas cada $10 \mathrm{~min}$. En aquellas cuyo muestreo era de menor frecuencia se interpolaba el valor. En particular, se aplicó este tratamiento a la temperatura de arrabio.

\section{TEMPERATURA SUBYACENTE}

El primer (y prácticamente constante) problema a resolver es: ¿A qué llamar temperatura del arrabio?. La respuesta a esta pregunta condiciona todo el proceso posterior y la validez o no de los resultados.

En principio, lo único que se puede medir es la temperatura del arrabio que sale por la piquera del horno y se ha de asociar cada temperatura con un instante determinado. Experimentalmente, se sabe que ésta varía dentro de una misma colada hasta $20^{\circ} \mathrm{C}$, siendo, en términos generales, más alta al final de la misma aunque todas las coladas no tienen la misma duración, que depende, entre otras causas, del diámetro que va adquiriendo la piquera de colada.

La gráfica de la temperatura del arrabio (Fig. 1) es una línea en forma de onda periódica cuyo periodo oscila entre 24 y $72 \mathrm{~h}$, sobre la que cabalga otra onda de mucho menor amplitud y mayor frecuencia.

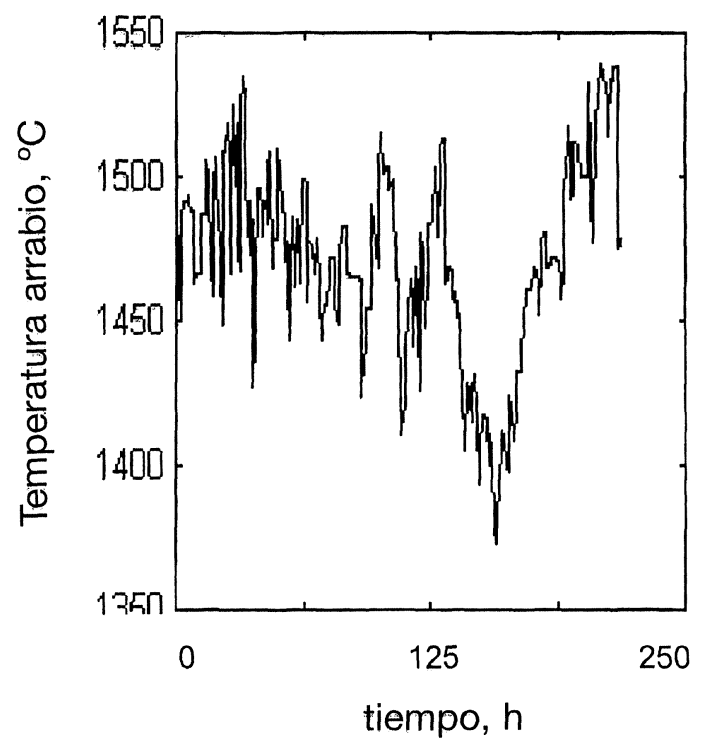

Figura 1. Temperaturas de colada.

Figure 1. Hot metal temperature.

(c) Consejo Superior de Investigaciones Científicas Licencia Creative Commons 3.0 España (by-nc)
Dicho de otra manera, en el horno alto existe una tendencia global de la temperatura del arrabio y, dentro de ésta, se producen oscilaciones limitadas a corto plazo. Esto sugiere que dentro del sistema termodinámico global del horno alto existe un subsistema que, aunque está básicamente relacionado con el sistema global, presenta oscilaciones locales de temperatura en función de determinadas circunstancias.

A la tendencia global de la temperatura del horno la llamaremos "temperatura subyacente" y su determinación en tiempo real y su predicción serán objetos de este trabajo.

\section{MODELO TEMODINAMICO}

El esquema teórico termodinámico más sencillo de un horno alto, que proporciona un comportamiento como el observado en la figura 1 (onda principal sumada a onda de rizado) es el de la figura 2 . Consiste en una fuente de calor que tiene una inercia térmica (capacidad calorífica) y que, asimismo, tendrá un límite máximo en cuanto a generación calorífica. Esta fuente está conectada a un receptor de calor principal con su (gran) inercia térmica. A su vez, ambos están conectados a un receptor secundario de mucha menor inercia térmica que el principal, dotado de un sumidero de calor. El receptor primario y, en menor medida, el secundario, tienen unas dimensiones físicas que provocan retardos entre el suministro de calor de la fuente y su expansión por todo el receptor primario hasta poder, a su vez, transmitir este calor al receptor secundario ${ }^{[1]}$ (Fig. 2).

El calor generado en la fuente fluiría hasta el sumidero a través de ambas conexiones. El receptor primario es el que tendría mayor inercia térmica y sería el sistema que da la temperatura "tendencia" o "subyacente" del horno y, por lo tanto, la temperatura "subyacente" del arrabio líquido. La conexión fuente-receptor secundario es la que introduciría el "rizado". La inercia térmica del

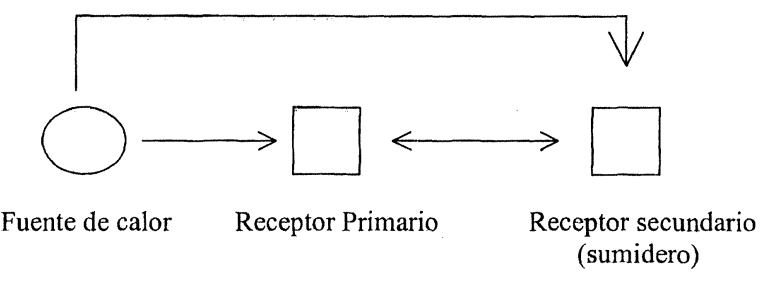

Figura 2. Esquema termodinámico.

Figure 2. Thermodynamic model.

Rev. Metal. Madrid 38 (2002) 243-248 http://revistademetalurgia.revistas.csic.es 
receptor secundario sería notablemente inferior a la del primario, esto es, de alrededor de un orden de magnitud.

En el receptor secundario (que en realidad es el subsistema de salida del arrabio), forma parte fundamental, aunque no única, la masa que tiene en ese momento el arrabio líquido, y se puede imaginar, por lo tanto, que su inercia térmica varíe con la cantidad que exista de dicho material, en cada momento. Dado que el calor que recibe de la fuente de calor sería, a grosso modo, constante, se podría suponer (aunque no se dispone de datos experimentales que lo puedan ratificar o descartar) que las variaciones de temperatura por unidad de tiempo y masa son mayores al final de cada colada.

Se considera que, la determinación y eventual predicción del comportamiento de la onda principal de la temperatura del arrabio, a la que se ha denominado temperatura "subyacente", es de gran interés porque es la que permite conocer la tendencia global, a medio y largo plazo, del alto horno, a diferencia de la temperatura instantánea, cuya rápida oscilación, debida a las características propias del proceso de salida del horno alto, no da información clara de la evolución la temperatura del horno alto.

Otro aspecto importante de este trabajo es el relacionado con el error admisible en la predicción. Para ello se fijó un critetio sencillo: comparar el error obtenido por el sistema predictivo a construir (en este caso será una red neuronal), con el error de una "predicción trivial", que es el etror cometido al considerar que la temperatura no va a sufrir variación desde el momento en que se hace la predicción hasta el instante predicho. Al cociente entre el segundo y el primero se le denominará "calidad del error". Cuanto mayor sea el cociente mejor será la predicción de la red neuronal. Cocientes menores o próximos a uno serán predicciones no válidas.

\section{METODOLOGÍA Y DESARROLLO DEL TRABAJO}

Como herramienta software informática se utilizó el paquete MatLab sobre un PC Pentium, tanto para el tratamiento de los datos, como para el desarrollo de la red neuronal y salidas gráficas.

Los datos suministrados por Aceralia constaban de 10.000 secuencias de numerosos parámetros, entre las que se encontraban las variables de soplado.

Se diseñó la red neuronal tomando como base un perceptrón multicapa con algoritmo de entre- namiento de Levenberg-Marquard ${ }^{[2]}$ y como entra= das las variables de soplado, además de la relación ore/coke y la velocidad de descenso de la carga con diferentes retrasos.

La ecuación del regresor (1) es de la forma:

$$
\begin{gathered}
\mathrm{Y}=\mathrm{F}(\mathrm{t}-1, \mathrm{t}-2 \ldots, \mathrm{t}-\mathrm{n}, \mathrm{O}-1, \ldots \mathrm{O}-\mathrm{n}, \mathrm{C}-1, \ldots, \\
\mathrm{C}-\mathrm{n}, \mathrm{H}-1, \ldots, \mathrm{H}-\mathrm{n}, \mathrm{A}-1 \ldots \mathrm{A}-\mathrm{n})
\end{gathered}
$$

siendo,

$\mathrm{t}-1, \ldots, \mathrm{t}-\mathrm{n} \rightarrow$ las temperaturas con difetentes re $=$ trasos $(13<\mathrm{n}<31)$

$\mathrm{O}-1, \ldots, \mathrm{O}-\mathrm{n} \rightarrow$ las inyecciones de oxigeno con $\mathrm{di}-$ ferentes retrasos $(13<\mathrm{n}<31)$

$\mathrm{C}-1, \ldots, \mathrm{C}-\mathrm{n} \rightarrow$ las inyecciones de carbón con diferetites retrasos $(13<\mathrm{n}<31)$

$\mathrm{H}-1, \ldots, \mathrm{H}-\mathrm{n} \rightarrow$ la humedad con diferentes retrasos $(13<\mathrm{n}<31)$

A-1...,A-n $\rightarrow$ el caudal de aire con diferentes tetrasos $(13<\mathrm{n}<31)$

$\mathrm{F}-1, \ldots, \mathrm{F}-\mathrm{n} \rightarrow$ relación $\mathrm{Fe} /$ coque con diferentes retrasos $(13<\mathrm{n}<31)$

$\mathrm{D}-1, \ldots, \mathrm{D}-\mathrm{n} \rightarrow$ velocidad descenso carga con diferentes retrasos $(13<\mathrm{n}<31)$

Se hicieron distintos ensayos vatiando, entre 14 y 30 , el número de retrasos de las variables introducidøs y, etitre 1 y 4 , el número de neuronas de la capa intermedia u oculta para observar los re= sultados. La configuración final óptima, que ya se adelantó, fue de 1 neurona en la capa intermedia para todas la hipótesis y un retraso de 14 secuencias en el intervalo de muestreo de 30 min en la mayoría de los casos, aunque en predicciones a mas de $10 \mathrm{~h}$ se mejoraba algo el error introduciendo (en función del tiempo de predicción) entre 20 y 30 secuencias de retraso.

Dada la gran inercia de un horno, para encontrar una relación efecto-causa en él, es necesario tomar en cada una de las variables de entrada periodoss que cubran varias horas, con un muestreo de intervalos de $10 \mathrm{~min}$, número de datos que era superior a las disponibilidades de memoria del ordenador. Para reducir el número de entradas, se aumentó el periodo de muestreo a $30 \mathrm{~min}$, con lo que el númeto de secuencias se redujo a la tercera parte.

Se utilizaron algo más de la mitad de los datos en el entrenamiento de la red (aprox., 1.800 secuencias) y el resto (aprox., 1.400), en la validación de la misma.

Inicialmente, se emplearon los datos con el único tratamiento de eliminación de errores 
(algoritmos hechos a medida para detectar inconsistencias), sin filtro matemático. Los resultados, con distintos tipos de estructuras de red neuronal, fueron poco alentadores incluso en previsiones a un plazo inferior a $2 \mathrm{~h}$. La razón es, posiblemente, lo poco previsible del proceso de sangrado del que depende la temperatura a corto plazo. Las rápidas e imprevisibles fluctuaciones a corto plazo de la temperatura del arrabio dan lugar a unos datos con excesiva oscilación, lo que impide encontrar un algoritmo matemático que pueda predecir el futuro en función de esos datos.

El mecanismo más usado en técnicas experimentales para evitar este problema es la aplicación de un filtro pasabajos ${ }^{[3]}$ a la señal problemática. Como es sabido, un filtro pasabajos es un método matemático para redondear la señal fijando una frecuencia máxima de salida.

La aplicación de filtros pasabajos ${ }^{[4]}$ de doble dirección a todos los datos (los de entrenamiento y los de validación), antes de ser introducidos en la red neuronal, mejoró la calidad de la predicción en, casi, un orden de magnitud. Sin embargo, esta técnica no se puede emplear para datos en tiempo real porque se necesita la totalidad de datos de la curva y, en un proceso en tiempo real, solo conocemos los datos del pasado, no los del futuro. La alternativa sería utilizar un filtro matemático de un solo sentido (que solo necesita los datos del pasado), pero tampoco es válida en algunos procesos en tiempo real como éste, porque produce un retraso de la "onda" un tiempo significativo y hace que los datos de previsión estén obsoletos antes de conocerse.

\section{FILTRO LIMITATIVO}

Para conseguir un filtrado eficaz, válido en tiempo real, se parte del esquema teórico del horno alto de la figura 2 y de las consideraciones del apartado 3 . Consiste en una fuente de calor conectada a dos receptores: uno, al que se denomina primario, que posee una gran inercia térmica y, otro, denominado secundario, con menor inercia térmica que el anterior. A su vez, ambos receptores disponen de una conexión entre sí.

La inercia térmica del sistema receptor primario implica que, para una fuente de calor con una potencia máxima, exista un incremento máximo de la temperatura en la unidad de tiempo, lo que, en una gráfica temperatura/tiempo, se traduce en que la pendiente no podría exceder de un valor predeterminado. Dadas las grandes dimensiones del horno, los tiempos de transmisión de las temperaturas de la fuente al receptor primario son apreciables. Esto, unido al aislamiento del horno, hace que las variaciones en los intercambios de calor tengan un límite, lo que se traduce en que los cambios de pendiente en la curva de la temperatura también lo tengan. Haremos hincapié en que todos estos factores son, en la práctica, constantes propias del horno, cuya variación, de producirse, sería lenta y a largo plazo, haciendo fácil su (re)cálculo y aplicación.

Estos dos principios, los de pendiente máxima y los de cambio máximo de pendiente, son los que se aplicaron para la elaboración de un filtro, que se denominó "limitativo", que permitiera conocer exclusivamente la temperatura subyacente y lo hiciera en tiempo real.

El filtro limitativo comprueba, en tiempo real, si el último valor de la temperatura suministrado produce, con respecto a los dos valores anteriores, una pendiente o un cambio de pendiente mayor que el preestablecido. De ser así, interpreta que es un valor de "rizado" y lo cambia al valor máximo permitido. El proceso se repite con los siguientes valores; en el caso de que encuentre un valor que no viole los máximos, lo dejará igual.

El empleo del filtro tiene otra ventaja adicional: elimina o limita el efecto de errores esporádicos en los datos.

Una vez establecido el tipo de filtro, el problema consiste en establecer el valor de la pendiente máxima y de la variación máxima de pendiente para la curva de temperatura. Debería ser un método empírico basado en los datos. Para establecer este criterio se ensayaron, sobre los datos suministrados, distintos filtros pasabajos de doble sentido, hasta encontrar uno que eliminaba la modulación sin modificar la onda básica. El filtro en este caso resultó ser de 1/10. Esta relación es probablemente cercana a la que existe entre las inercias del sistema global y el subsistema "salida". En la señal que produjo este filtro, se examinaron los valores máximos de la pendiente y del cambio de ésta. Estos valores se introdujeron en el filtro "limitativo" y se trazó, con este último, la curva de temperatura/ tiempo. La curva resultante era muy similar a la que producía el filtro estándar de doble dirección (Fig. 3).

\section{RESULTADOS}

Una vez elaborado el filtro limitativo de este horno alto, se aplicó a los datos (entrenamiento y 

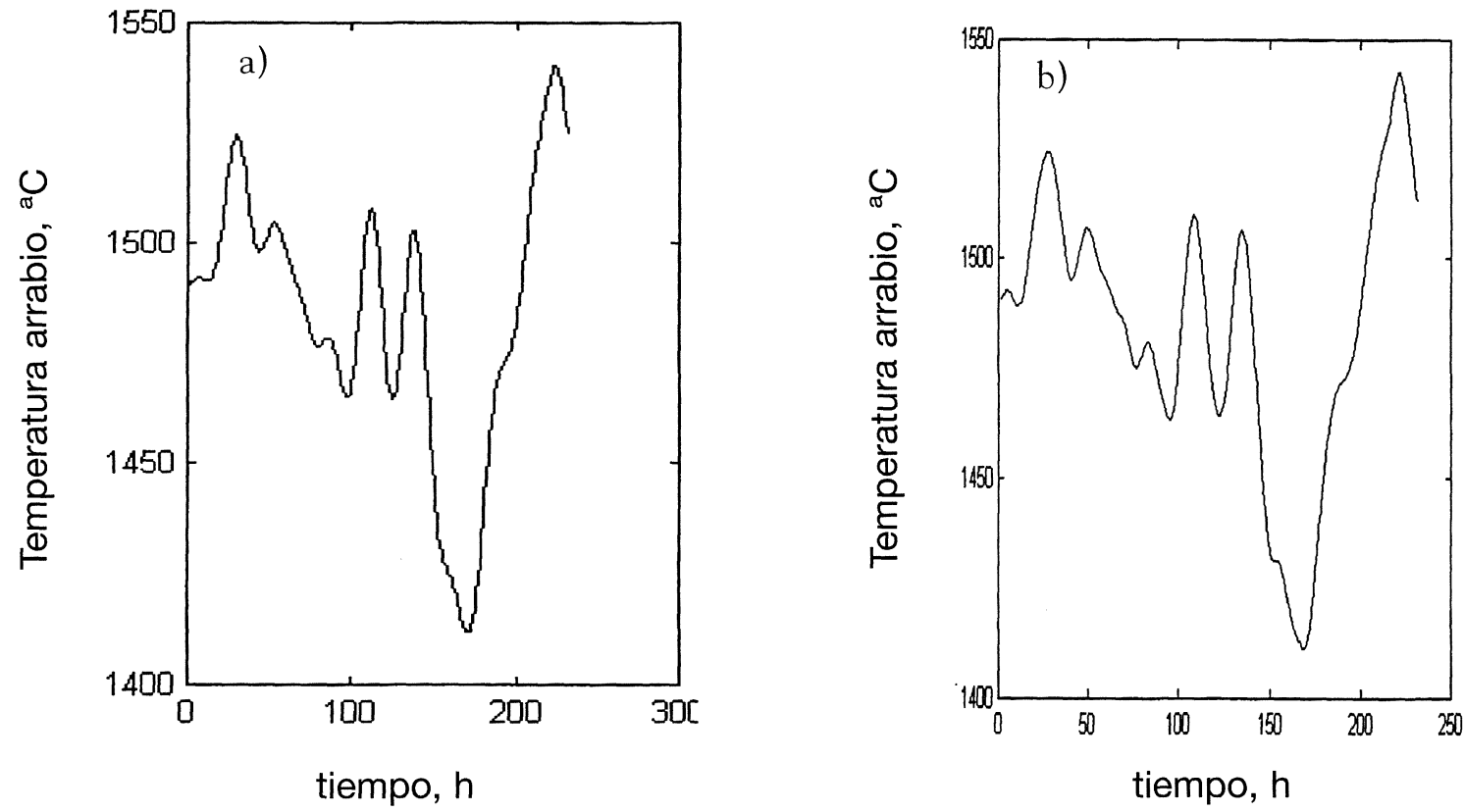

Figura 3. Temperaturas filtradas. a) Con filtro de doble sentido. b) Con filtro limitativo.

Figure 3. Filtered temperatures. a) By a double sense filter. b) By a limiter filter.

validación) de la temperatura de salida del arrabio líquido, antes de introducirlos en la red neuronal, y se obtuvieron los resultados que se reflejan en la tabla I y la figura 4.

La tabla I muestra, en la primera columna, el intervalo de tiempo a predecir, en h; en la segunda, los errores medios absolutos de la temperatura, en ${ }^{\circ} \mathrm{C}$; en la tercera, el error de la predicción trivial y, en la ultima columna, las "calidades de error" (definidas al final del apartado 2). La validez de la predicción se manifiesta con más claridad mediante este último parámetro.

Como se puede observar, el error en la predicción es prácticamente nulo hasta las $9 \mathrm{~h}$ de predicción, siendo entonces la calidad de error 34,1, incrementándose rápidamente hasta las $18 \mathrm{~h}$. A partir de este periodo de tiempo, el valor predicctivo de la red neuronal es ya muy escaso (calidad de error 1,4).

$\mathrm{Al}$ examinar los pesos de las distintas entradas de la red neuronal, se encontró que los valores correspondientes a las entradas de soplado eran despreciables, por lo que se eliminaron dichas entradas de la red, con resultados finales análogos.

\section{CONSIDERACIONES FINALES}

El filtro "limitativo", además de instrumento previo para tratar los datos que posteriormente servirán de entrada a la red neuronal, puede ser utilizado, directamente, como "termómetro de tendencia" del horno alto.

Respecto a la estructura final de las entradas de la red neuronal, puede resultar extraño que prescinda de las entradas que no sean la temperatura para encontrar una predicción válida a medio plazo. Lo más probable es que esta aparente paradoja sea el resultado de dos razones concurrentes. $\mathrm{La}$

Tabla I. Errores de la predicción

Table I. Prediction errors

\begin{tabular}{cccc}
\hline Horas & Error $/{ }^{\circ} \mathrm{C}$ & Predic. trivial & Calidad de error \\
\hline 9 & 0,644 & 21,98 & 34,1 \\
10 & 1,285 & 22,30 & 17,35 \\
11 & 2,161 & 22,36 & 10,3 \\
12 & 3,48 & 22,40 & 6,4 \\
13 & 4,915 & 22,50 & 4,5 \\
14 & 7,301 & 22,75 & 3,11 \\
15 & 9,945 & 23,26 & 2,3 \\
16 & 11,47 & 23,83 & 2,07 \\
17 & 13,00 & 24,33 & 1,87 \\
18 & 15,41 & 24,77 & 1,60 \\
19 & 16,92 & 25,26 & 1,49 \\
20 & 17,81 & 25,84 & 1,45 \\
21 & 18,29 & 26,40 & 1,45 \\
22 & 18,56 & 26,92 & 1,45 \\
\hline
\end{tabular}


Predición mediante redes neuronales de la temperatura de arrabio de un horno alto. Temöperatura subyacente de arrabio C. CANTERA, J. JimÉNEZ, I. VARELA y A. Formoso
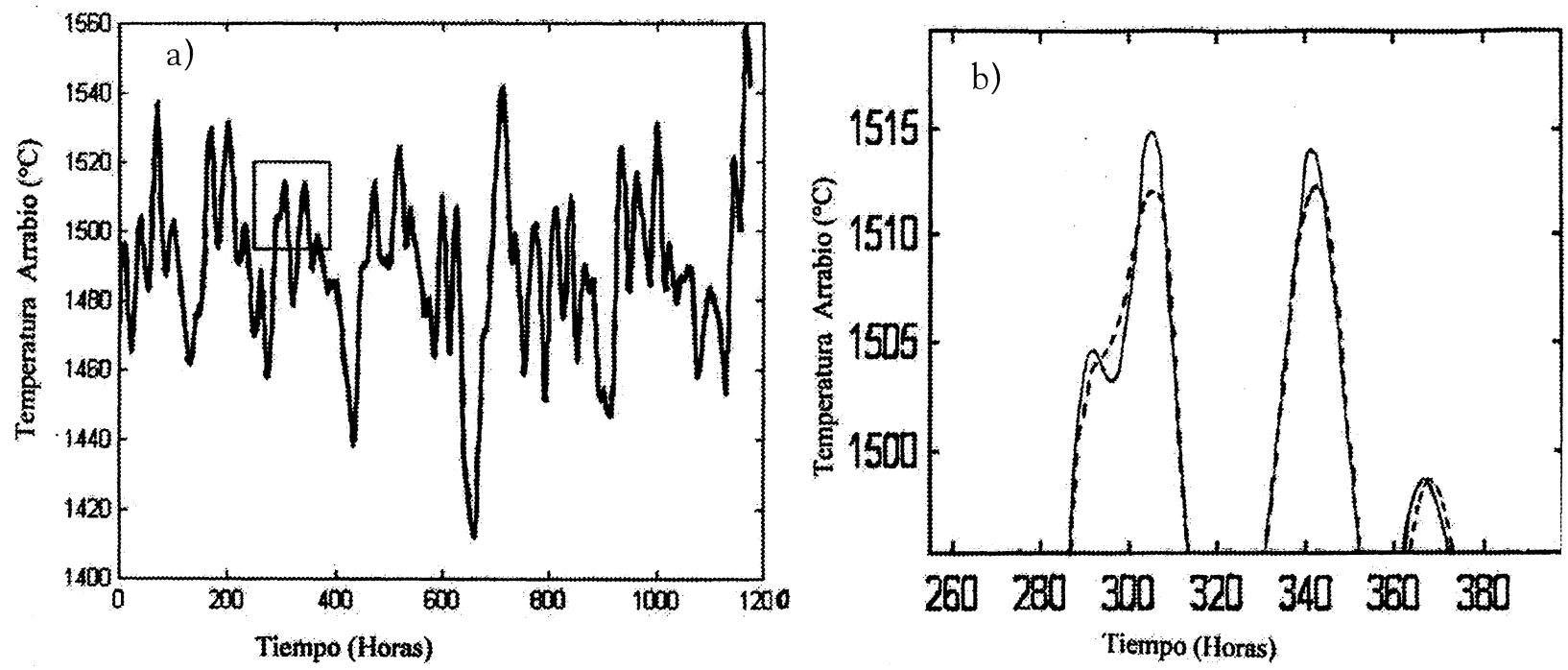

Figura 4. Temperatura medida (línea continua) y predicción a $10 \mathrm{~h}$ (línea discontinua). b) Ampliación del recuadro insertado en a).

Figure 4. a) Measured temperature (continuous line) and $10 \mathrm{~h}$ prediction (dotted line). b) Enlargement of the box giving in a).

primera, que la inercia térmica del horno es tân grande que éste, a corto y medio plazo, impone en gran medida su propia dinámica. La segunda, que las acciones del operador de lâ planta son siempre las mismas ante una situación determinada del horno alto, es decir, que dada una secuencia de temperaturas determinada se puede predecir la actuación del operador y, por tanto, la subsecuente respuesta del horno. Esto significa que la acción del operador se puede describir con una función matemática que depende de la historia de las últimas temperaturas. La reacción del horno es función de estas acciones y, por lo tanto, de la historia reciente de las temperaturas. Dicho de otra manera, la red ha encontrado a medio plazo las curvas de respuesta del sistema horno-operador.

Una posible continuación de este trabajo sería realizar un estudio a largo plazo, cuya utilidad final podría ser el control automatizado de un horno alto. Es obvio que, en este caso, el valor de las variables de soplado ha de ser determinante, aunque el planteamiento del entrenamiento de la red neuronal tendría que ser diferente.

\section{Agradecimientos}

Los autores agradecen a la Comunidad Europea del Carbón ỳ el Acero (contrato 7210-AA-940) y a la CICYT (MAT96-1776) la financiación económica del proyecto "Investigation of Fuzzy Logic and Neural Network Based Strategies for Control Optimisation of Iron Process", dentro del cual se enmarca el presente trabajo, así como a la empresa Aceralia, y más concretamente a los Drs. B. Fernández y J. Sainz, por su colaboración.

\section{REFERENCIAS}

[1] L. LJung, System Identification, Prentice Hall, 1999, pp. 458 475.

[2] M. Hagan, H. Demuth and M. Beale, Neural Network Design, PWS Publishing Company, 1995, pp. 11,4-11,5.

[3] J.S. BENDAT and A.G. PIERSOL, Random data: analysis and measurament procedures, Wiley=Interscience, 1971, p. 291.

[4] Matlab, Signal Processing Toolbox. User's Guide,v.5, The Math Works Inc., 2000, p. 5.2. 